\title{
Singular Continuous Measures in Scattering Theory
}

\author{
D. B. Pearson
}

Department of Applied Mathematics, University of Hull, Hull HU6 7RX, England

\begin{abstract}
Examples are presented of potentials $V$ for which $-\frac{d^{2}}{d r^{2}}+V(r)$ in $L^{2}(0, \infty)$ has singular continuous spectrum, and the physical interpretation is discussed.
\end{abstract}

\section{Introduction}

Corresponding to the decomposition of a measure $\mu$ on $\mathbb{R}$ into pure point, absolutely continuous (with respect to Lebesgue measure) and singular continuous parts, in Quantum Mechanics one has a canonical decomposition of the underlying Hilbert Space $\mathscr{H}$ into the direct sum of mutually orthogonal subspaces $M_{p}(H)$, $M_{\text {a.c. }}(H)$ and $M_{\text {s.c. }}(H)$ [1] defined by the total Hamiltonian operator $H$.

In most cases, $M_{p}(H)$ may be taken to be the subspace spanned by the bound states of the system, and $M_{\text {a.c. }}(H)$ the subspace of scattering states (i.e. states which, in the limit as $t \rightarrow \pm \infty$, are asymptotically far from the scattering centre). For a more detailed discussion see [2,3]. For a potential which is highly singular, and which gives rise to absorption at local singularities, $M_{\text {a.c. }}(H)$ may itself be decomposed into the subspaces respectively of scattering states and of states which are asymptotically absorbed [4].

The remaining subspace $M_{\text {s.c. }}(H)$ has usually been supposed to admit no physical interpretation (see for example [5], p. 23). Indeed, in non-relativistic potential scattering theory, considerable attention has been given to the derivation of conditions under which $M_{\text {s.c. }}(H)=\{0\}$. From the extensive literature on this subject we refer to the work of Weidmann [6] and Lavine [7, 8]. Weidmann has proved the absence of singular continuous spectrum for (non-singular) spherical potentials $V=V_{1}+V_{2}$, with $V_{1}$ of bounded variation and $V_{2}$ of short range. Lavine has proved the same result for potentials satisfying a so called "no bump" condition $\frac{r}{2} \frac{d V}{d r}+V<$ const. (These results apply in greater generality, e.g. to nonspherical potentials.) By classical analogy, this condition means that an incoming particle will encounter no effective obstacle and will ultimately recede to infinity having been scattered by the potential. 
These and other results suggest that one may associate singular continuous spectrum with potentials having infinitely many "bumps", provided their height does not diminish too rapidly with distance. The present paper is devoted to justifying this view by presenting a wide range of potentials giving rise to singular continuous spectrum, and, finding a physical interpretation for them.

It is already known from the inverse method of scattering theory [9] that singular continuous spectrum can occur. Aronszajn [10] has constructed an example giving rise either to a dense set of eigenvalues or to singular continuous spectrum, depending on the boundary conditions. However, in the inverse method one arrives at the potential indirectly from the solution of an integral equation. One has no control, for example, of the behaviour of the potential for large $r$, and a physical interpretation is lacking. We shall start from the potential and determine the spectrum from estimates of the associated spectral function. (For definition and properties of the spectral function associated with a second order differential operator see [11].)

Using the Cantor measure [12] as a starting point, Section 2 concerns the generation of singular continuous measures from limits of absolutely continuous measures; the measures here do not necessarily come from any problem with differential operators, though this provides the motivation. The main results are Theorem 1 and its Corollary, which are applied in Section 3 to the spectral measure corresponding to a potential made up of an infinite sequence of bumps, their separation increasing rapidly with distance. The potential is spherically symmetric (or one dimensional), bounded, and locally non-singular. It is even possible to have $\lim V(r)=0$, in which case the condition for singular continuous spectrum is (roughly) $\sum_{n=1}^{\infty} g_{n}^{2}=\infty$, where $g_{n}$ is the height of the $n$ 'th bump. (The shape of individual bumps and the size of "coupling constant" are both arbitrary.) This is exactly the condition for the particle, after possibly a large number of transmissions and reflections at successive bumps, ultimately with probability 1 to be reflected back to a neighbourhood of the origin. Although the particle will subsequently be at a large distance from the origin, it will return arbitrarily often. (C.F. in the classical theory of stochastic processes [13], the property of recurrence.) This semi-classical analogy is justified in Section 4 by an analysis in terms of wave-packets. Thus for these potentials giving rise to singular continuous spectrum we have the physical interpretation that they are to be regarded as presenting to the particle a totally reflecting barrier, into which the particle may penetrate arbitrarily far but must eventually be reflected. (On a more mathematical level, let us note that, for non-singular long range potentials, compactness arguments imply [2] that for a state in the continuum subspace to move asymptotically, with probability 1 , to infinity, one has the necessary and sufficient condition $w$-lim $e^{-i H t} f=0$. This asymptotic condition, which follows from the Riemann-Lebesgue Lemma under the assumption $M_{\text {s.c. }}(H)=\{0\}$, need not apply in general. However, for $f \in M_{c}(H)$, the mean-squared probability of finding the particle in any fixed bounded region converges to zero.)

In Section 5 we present a more general analysis based on the asymptotic behaviour of products of transfer matrices. This enables us to construct examples of singular continuous spectrum arising from perturbations of both periodic 
potentials and (highly singular) short range potentials. For example, to any $L^{1}(0, \infty)$ potential may be added an arbitrarily small (in the sense of $L^{\infty}$ norm) perturbation converting the spectrum from absolutely continuous to singular continuous. From the more general point of view of Section 5, singular continuous spectrum appears in a wide variety of contexts as a "small" and not particularly pathological perturbation of absolutely continuous spectrum. Potentials which give rise to this phenomenon are, in some sense, "everywhere dense" in the set of all potentials.

\section{Generation of Singular Continuous Measures}

One of the classic examples of a singular continuous measure is the LebesgueStieltjes measure generated by the Cantor function [12]. The support of this measure is the Cantor set $C$, consisting of all points $x$ in $[0,1]$ have a decimal expansion to the base 3 ,

$$
x=0 \cdot x_{1} x_{2} x_{3}, \ldots,\left(\text { or } x=\sum_{i=1}^{\infty} \frac{x_{i}}{3^{i}}\right)
$$

in which each $x_{i}$ is either 0 or 2 . The Cantor Function may be defined on $[0,1]$ by

$$
\psi(x)=\sum_{i=1}^{\infty} \frac{x_{i}}{2^{i+1}}, \quad x \in C,
$$

and

$$
\psi(x)=\sum_{1 \leqq i<n} \frac{x_{i}}{2^{i+1}}+\frac{1}{2^{n}}, \quad x \notin C,
$$

where $n(x)$ is the smallest integer for which $x_{n}=1 ; \psi$ is then continuous and nondecreasing and generates a singular continuous measure $\mu$ on the Borel subsets of $[0,1]$.

An alternative approach, which we shall adopt, is to define a function $f$, periodic with period 1 , such that

$$
\begin{array}{rlrl}
f(x)=1 & & 0 \leqq x \leqq \frac{1}{3} \\
& =0 & & \frac{1}{3}<x<\frac{2}{3} \\
& =1 & & \frac{2}{3} \leqq x \leqq 1 .
\end{array}
$$

Then the characteristic function of the Cantor set is given by

$$
\chi(x)=\prod_{k=1}^{\infty} f\left(3^{k-1} x\right),
$$

and the Cantor function may be expressed in the form

$$
\psi(x)=\lim _{n \rightarrow \infty} \int_{0}^{x} d x \prod_{k=1}^{n} f_{k}(x),
$$

where

$$
f_{k}(x)=\frac{3}{2} f\left(3^{k-1} x\right)
$$

For any subinterval $\Sigma$ of $[0,1]$ we have, then,

$$
\mu(\Sigma)=\lim _{n \rightarrow \infty} \int_{\Sigma} d x \prod_{k=1}^{n} f_{k}(x) .
$$


There are pitfalls for the unwary! Note that (1) does not hold in general if $\Sigma$ is an arbitrary Borel subset. Moreover, one cannot interchange limit and integral in (1), and indeed

$$
\begin{aligned}
\prod_{k=1}^{\infty} f_{k}(x) & =\infty, \quad x \in C \\
& =0 \quad x \notin C .
\end{aligned}
$$

The idea of generating in this way a singular continuous measure $\mu$ from a limiting sequence of absolutely continuous measures may be considerably extended. From this more general point of view, the principal properties of the $f_{k}$ which we shall retain are

a) mean $\left(f_{k}\right)=\bar{f}_{k} \equiv \int_{0}^{1} f_{k}(x) d x=1$, and

b) as $k \rightarrow \infty$, the $f_{k}$ oscillate increasingly rapidly. star.)

(Throughout this paper, means are denoted by bar and complex conjugates by

We shall, however, differ from Cantor measure in that the $f_{k}$ will be taken to be continuous (even differentiable) and bounded away from zero, this being more appropriate to applications in potential scattering.

With the above properties in mind we can now state

Theorem 1. Let the functions $f_{n}(k, y)(\alpha \leqq k \leqq \beta,-\infty<y<\infty, n=1,2, \ldots)$ be periodic in $y$, with period $c$, continuously differentiable, and satisfy

i) $f_{n}(k, y) \geqq$ const $>0$,

ii) $\overline{f_{n}}(k) \equiv \frac{1}{c} \int_{0}^{c} f_{n}(k, y) d y=1$,

iii) $\sum_{n=1}^{\infty}-m_{n}(k)=+\infty, \quad \alpha \leqq k \leqq \beta$,

where

$$
m_{n}(k)=\overline{\log }\left(f_{n}(k, y)\right)=\frac{1}{c} \int_{0}^{c} \log f_{n}(k, y) d y .
$$

iv) For $N$ sufficiently large, $f_{n}(k, N k)$ is an analytic function of $k, \alpha \leqq k \leqq \beta$.

Given a sequence $\left\{N_{i}\right\}, i=1,2,3, \ldots$, of increasing positive numbers with $\lim _{i \rightarrow \infty} N_{i}=\infty$, define the Lebesgue-Stieltjes measures $\mu_{n}$ by

$$
\mu_{n}(\Sigma)=\int_{\Sigma} d k \prod_{i=1}^{n} f_{i}\left(k, N_{i} k\right),
$$

for every subinterval $\Sigma$ of $[\alpha, \beta]$.

Then the sequence $\left\{N_{i}\right\}$ may be chosen such that $\lim _{n \rightarrow \infty} \mu_{n}(\Sigma)=\mu(\Sigma)$ exists for every subinterval $\Sigma$ of $[\alpha, \beta]$ and defines a singular continuous Lebesgue-Stieltjes measure on the Borel subsets of $[\alpha, \beta]$.

The proof is in five stages. 
Step I. Given any $\varepsilon>0, \exists N_{0}$ (which may depend on $n$ ) such that, for every subinterval $\Sigma$ of $[\alpha, \beta]$,

$$
\left|\int_{\Sigma} f_{n}(k, N k) d k-\mu_{0}(\Sigma)\right|<\varepsilon
$$

for $N>N_{0}$, where $\mu_{0}$ denotes Lebesgue measure.

Proof. Let $g_{n}(k, y)=f_{n}(k, y)-1$, so that $\int_{0}^{c} g_{n}(k, y) d y=0$. Choose $N_{0}$ sufficiently large that $\frac{c}{N_{0}} \max _{k, y}\left|g_{n}(k, y)\right|<\varepsilon / 2$, and

$$
\left|k_{1}-k_{2}\right| \leqq \frac{c}{N_{0}} \Rightarrow\left|g_{n}\left(k_{1}, y\right)-g_{n}\left(k_{2}, y\right)\right|<\frac{\varepsilon}{2}(\beta-\alpha)^{-1}
$$

Now subdivide $\Sigma$ into $r$ intervals $I_{i}$, with $\mu_{0}\left(I_{i}\right)=\frac{c}{N}(i=1,2, \ldots(r-1))$ and $\mu_{0}\left(I_{r}\right) \leqq c / N_{0}$.

(If $\mu_{0}(\Sigma) \leqq c / N$, there will be only a single interval.)

If $N \geqq N_{0}$, we have

$$
\left|\int_{I_{r}} g_{n}(k, N k) d k\right|<\frac{\varepsilon}{2},
$$

and

$$
\left|\int_{I_{i}}\left[g_{n}(k, N k)-g_{n}\left(k_{i}, N k\right)\right] d k\right|<\frac{\varepsilon}{2}(\beta-\alpha)^{-1} \mu_{0}\left(I_{i}\right)
$$

provided $i<r$ and $k_{i} \in I_{i}$.

Moreover,

$$
\int_{I_{i}} g_{n}\left(k_{i}, N k\right) d k=\frac{1}{N} \int_{0}^{c} g_{n}\left(k_{i}, y\right) d y=0,
$$

so that summing over all subintervals $I_{i}$ we obtain

$$
\left|\int_{\Sigma} g_{n}(k, N k) d k\right| \leqq \frac{\varepsilon}{2}+\frac{\varepsilon}{2}=\varepsilon .
$$

Step II. Given $\left\{N_{i}\right\}(i=1,2, \ldots, n)$ and $\varepsilon>0, \exists N_{0}$ such that, for every subinterval $\Sigma$ of $[\alpha, \beta],\left|\mu_{n+1}(\Sigma)-\mu_{n}(\Sigma)\right|<\varepsilon$ for $N_{n+1} \geqq N_{0}$.

Proof.

$$
\mu_{n+1}(\Sigma)-\mu_{n}(\Sigma)=\int_{\Sigma} d k g_{n+1}\left(k, N_{n+1} k\right) \prod_{i=1}^{n} f_{i}\left(k, N_{i} k\right) .
$$

Writing

$$
g_{n+1}\left(k, N_{n+1} k\right)=\frac{d}{d k} \int_{a_{1}}^{k} g_{n+1}\left(k, N_{n+1} k\right) d k,
$$


where $\inf \Sigma=a_{1}$, and integrating by parts, the result follows from I, since both $\prod_{i=1}^{n} f_{i}\left(k, N_{i} k\right)$ and $\frac{d}{d k} \prod_{i=1}^{n} f_{i}\left(k, N_{i} k\right)$ are bounded.

Step III. The sequence $\left\{N_{i}\right\}$ may be chosen such that, for every subinterval $\Sigma$ of $[\alpha, \beta], \mu(\Sigma)=\lim _{n \rightarrow \infty} \mu_{n}(\Sigma)$ exists and defines a continuous measure.

Proof. From II, we can take each $N_{n+1}$ sufficiently large (in comparison with the $N_{i}$ for $\left.i \leqq n\right)$ that the series $\sum_{i=1}^{\infty}\left(\mu_{i+1}(\Sigma)-\mu_{i}(\Sigma)\right)$ is absolutely and uniformly convergent for subintervals $\Sigma$. Hence $\mu(\Sigma)$ exists, and defines a Lebesgue-Stieltjes measure. If we now take $n$ such that $\left|\mu_{n}(\Sigma)-\mu(\Sigma)\right|<\varepsilon / 2$, for given $\varepsilon>0$ we can find $\delta>0$ such that $\mu_{0}(\Sigma)<\delta \Rightarrow \mu_{n}(\Sigma)<\varepsilon / 2$ (since $\mu_{n}$ is absolutely continuous).

Hence $\mu_{0}(\Sigma)<\delta \Rightarrow \mu(\Sigma)<\varepsilon$, and the measure $\mu$ is continuous [i.e. $\mu([\alpha, \lambda))$ is a continuous function of $\lambda$.]

Step IV. Given $\left\{N_{i}\right\} \quad(i=1,2, \ldots, m), \quad$ and $\quad \varepsilon, c^{\prime}>0, \quad \exists n>m$ and $\left\{N_{i}\right\}$ $(i=m+1, m+2, \ldots, n)$ such that

$$
\mu_{0}\left\{k ; \prod_{i=1}^{n} f_{i}\left(k, N_{i} k\right)>c^{\prime}\right\}<\varepsilon .
$$

Proof. Let $h_{n}(k, y)=\log f_{n}(k, y)=\log \left(1+g_{n}(k, y)\right)$, so that from (2) we have $\overline{h_{n}}(k)=m_{n}(k)$. First observe that from the inequality

$$
\log (1+x) \leqq x \quad(-1<x<\infty)
$$

we have

$$
h_{n}(k, y) \leqq g_{n}(k, y),
$$

so that ii) $\Rightarrow \overline{h_{n}}=m_{n} \leqq 0$.

Since the $m_{i}(k)$ are continuous, iii $) \Rightarrow \sum_{i=1}^{\infty}-m_{i}(k)=+\infty$ uniformly in $k$, by a compactness argument. So without less of generality we can assume

$$
\sum_{i=m+1}^{n}-m_{i}(k) \geqq \text { const }>0 .
$$

We further take $n$ sufficiently large that

$$
\frac{1}{2} \sum_{i=m+1}^{n} m_{i}(k)<\log \left(\frac{c^{\prime}}{M}\right),
$$

where

$$
M=\sup _{k} \prod_{i=1}^{m} f_{i}\left(k, N_{i} k\right) .
$$


Now

$$
\begin{aligned}
& \mu_{0}\left\{k ; \prod_{i=1}^{n} f_{i}\left(k, N_{i} k\right)>c^{\prime}\right\} \leqq \mu_{0}\left\{k ; \prod_{i=m+1}^{n} f_{i}\left(k, N_{i} k\right)>c^{\prime} / M\right\} \\
& =\mu_{0}\left\{k ; \sum_{i=m+1}^{n} h_{i}\left(k, N_{i} k\right)>\log \left(\frac{c^{\prime}}{M}\right)\right\} \leqq \mu_{0}\left\{k ; \sum_{i=m+1}^{n} h_{i}\left(k, N_{i} k\right)>\frac{1}{2} \sum_{i=m+1}^{n} m_{i}(k)\right\} \\
& \leqq \int_{\alpha}^{\beta} d k\left(\frac{\left[\sum_{i=m+1}^{n}\left(h_{i}\left(k, N_{i} k\right)-m_{i}(k)\right)\right]^{2}}{\left[\frac{1}{2} \sum_{i=m+1}^{n} m_{i}(k)\right]^{2}}\right)
\end{aligned}
$$

Writing $\overline{h_{i}^{2}}(k)=\frac{1}{c} \int_{0}^{c} h_{i}^{2}(k, y) d y$, we have

$$
h_{i}^{2}\left(k, N_{i} k\right)=\overline{h_{i}^{2}}(k)+\frac{d}{d k} \int_{\alpha}^{k}\left[h_{i}^{2}\left(k, N_{i} k\right)-\overline{h_{i}^{2}}(k)\right] d k,
$$

and as in II above we may integrate by parts to show that, for large $\left\{N_{i}\right\}(i=m+1$, $m+2, \ldots, n)$, and fixed $n$,

$$
\int_{\alpha}^{\beta} d k\left(\frac{\left[\sum_{i=m+1}^{n} h_{i}^{2}\left(k, N_{i} k\right)\right]}{\left[\frac{1}{2} \sum_{i=m+1}^{n} m_{i}(k)\right]^{2}}\right)
$$

becomes arbitrarily close to

$$
\int_{\alpha}^{\beta} d k\left(\frac{\left[\sum_{i=m+1}^{n} \overline{h_{i}^{2}}(k)\right]}{\left[\frac{1}{2} \sum_{i=m+1}^{n} m_{i}(k)\right]}\right) .
$$

[Note that the $m_{i}(k)$ are continuously differentiable.] Integrating with respect to $x$ the inequality

$$
\frac{\log (1+x)}{(1+x)} \leqq \frac{x}{(1+x)}
$$

we have

$$
[\log (1+x)]^{2} \leqq 2[x-\log (1+x)] \quad(0 \leqq x<\infty),
$$

which for $x \geqq$ const $>-1$ implies, for some $K>0$,

$$
[\log (1+x)]^{2} \leqq K[x-\log (1+x)] .
$$

Hence $\left[h_{i}(k, y)\right]^{2} \leqq k\left[g_{i}(k, y)-h_{i}(k, y)\right]$, which on integrating with respect to $y$ becomes

$$
\overline{h_{i}^{2}}(k) \leqq-K m_{i}(k) .
$$


We take $n$ sufficiently large that

$$
\int_{\alpha}^{\beta} d k\left(\frac{\left[\sum_{i=m+1}^{n} \overline{h_{i}^{2}}(k)\right]}{\left[\frac{1}{2} \sum_{i=m+1}^{n} m_{i}(k)\right]^{2}}\right) \leqq \frac{-4 K}{\sum_{i=m+1}^{n} m_{i}(k)}<\varepsilon / 4,
$$

and the $\left\{N_{i}\right\}(i=m+1, m+2, \ldots, n)$ sufficiently large that

$$
\int_{\alpha}^{\beta} d k\left(\frac{\sum_{i=m+1}^{n} h_{i}^{2}\left(k, N_{i} k\right)}{\left[\frac{1}{2} \sum_{i=m+1}^{n} m_{i}(k)\right]^{2}}\right) \leqq \frac{\varepsilon}{4}+\frac{\varepsilon}{4}=\varepsilon / 2 .
$$

In the same way we can take each $N_{i}$ sufficiently large, in comparison with the preceding $\left\{N_{j}\right\}$ that

$$
\int_{\alpha}^{\beta} d k\left(\frac{\sum_{i>j}\left[h_{i}\left(k, N_{i} k\right)-m_{i}(k)\right]\left[h_{j}\left(k, N_{j} k\right)-m_{j}(k)\right]}{\left[\frac{1}{2} \sum_{i=m+1}^{n} m_{i}(k)\right]^{2}}\right)
$$

becomes arbitrarily small, and each $N_{i}$ sufficiently large that

$$
\begin{aligned}
& \int_{\alpha}^{\beta} d k\left(\frac{\sum_{i=m+1}^{n}\left[-2 m_{i}(k) h_{i}\left(k, N_{i} k\right)+m_{i}^{2}(k)\right]}{\left[\frac{1}{2} \sum_{i=m+1}^{n} m_{i}(k)\right]^{2}}\right) \\
& \leqq \int_{\alpha}^{\beta} d k\left(\frac{\sum_{i=m+1}^{n}\left[-2 m_{i}(k)\left(h_{i}\left(k, N_{i} k\right)-m_{i}(k)\right)\right]}{\left[\frac{1}{2} \sum_{i=m+1}^{n} m_{i}(k)\right]^{2}}\right)
\end{aligned}
$$

has an arbitrarily small upper bound. Bounding these terms by $\varepsilon / 2$ we have finally, for suitable $\left\{N_{i}\right\}$,

$$
\mu_{0}\left\{k ; \prod_{i=1}^{n} f_{i}\left(k, N_{i} k\right)>c^{\prime}\right\} \leqq \varepsilon / 2+\varepsilon / 2=\varepsilon .
$$

Step $V$. The measure $\mu$ is singular, for suitable $\left\{N_{i}\right\}$.

Proof. Given $\varepsilon>0$, let

$$
S_{\varepsilon}=\left\{k ; \prod_{i=1}^{n} f_{i}\left(k, N_{i} k\right) \geqq \frac{\varepsilon}{(\beta-\alpha)}\right\},
$$

and use IV above to choose $\left\{N_{i}\right\}(i=1,2, \ldots, n)$ and $n$ such that $\mu_{0}\left(S_{\varepsilon}\right)<\varepsilon$. 
Since iv $) \Rightarrow \prod_{i=1}^{n} f_{i}\left(k, N_{i} k\right)$ is analytic, the equation $\prod_{i=1}^{n} f_{i}\left(k, N_{i} k\right)=\varepsilon /(\beta-\alpha)$ is either an identity or is satisfied at only a finite number of points, so that $S_{\varepsilon}$ consists of a finite number $r$ of intervals. (This is the only use of analyticity in the proof of the theorem.) In choosing $\left\{N_{i}\right\}$ for $i>n$, use III above to ensure that, for every subinterval $\Sigma$ of $[\alpha, \beta],\left|\mu_{n}(\Sigma)-\mu(\Sigma)\right|<\varepsilon / r$. It follows that $\left|\mu_{n}\left(S_{\varepsilon}\right)-\mu\left(S_{\varepsilon}\right)\right|<\varepsilon$.

Again,

$$
\mu_{n}\left(S_{\varepsilon}\right)=\mu_{n}([\alpha, \beta])-\mu_{n}\left\{k ; \prod_{i=1}^{n} f_{i}\left(k, N_{i} k\right)<\frac{\varepsilon}{(\beta-\alpha)}\right\} \geqq \mu_{n}([\alpha, \beta])-\varepsilon
$$

on using (3).

Hence $\mu\left(S_{\varepsilon}\right) \geqq \mu([\alpha, \beta])-3 \varepsilon$.

Continuing in this way, we may choose the $\left\{N_{i}\right\}$ such as to construct a sequence $\left\{S_{\varepsilon}\right\}$ of subsets of $[\alpha, \beta]$, with $\varepsilon \rightarrow 0$, for which $\lim _{\varepsilon \rightarrow 0} \mu_{0}\left(S_{\varepsilon}\right)=0$, whereas

$\lim _{\varepsilon \rightarrow 0} \mu\left(S_{\varepsilon}\right)=\mu([\alpha, \beta])$.

It follows that $\mu$ is singular with respect to Lebesgue measure, and this concludes the proof of the theorem.

It will be noticed that the proof of Theorem 1 has somewhat a probabilistic flavour. For large $N_{i}, h_{i}\left(k, N_{i} k\right)$ behaves in many respects like $h_{i}(k, u)$, where $u$ represents the value of a random variable uniformly distributed in an interval of length $c$. For large $N_{i}$ and $N_{j} / N_{i}$ the "independence" of $h_{i}\left(k, N_{i} k\right)$ and $h_{j}\left(k, N_{j} k\right)$ then follows from Riemann-Lebesgue type arguments and Step IV follows the standard proof of the weak law of large numbers to show that $\sum_{n=1}^{\infty} h_{n}=-\infty$ "with probability one". The singularity of $\mu$ is a consequence of the fact that, whereas $\prod_{i=1}^{n} f_{i}\left(k, N_{i} k\right)$ converges to zero in Lebesgue measure as $n \rightarrow \infty$, the limit vanishes only on a set of $\mu$-measure zero. (For convergence in measure and related concepts see [14], Chapter 7. Convergence in measure implies the existence of a subsequence converging almost everywhere.)

The nature of the measure $\mu$ may also be determined in the case $\sum_{n=1}^{\infty}-m_{n}(k)<\infty$. We assume the convergence to be uniform, and retain all other conditions of Theorem 1 . In that case $\prod_{i=1}^{n} f_{i}\left(k, N_{i} k\right)$ is found to converge to a finite but non-zero limit both in Lebesgue measure and in $\mu$-measure. We omit detailed proofs and observe only that (again for suitable $\left.\left\{N_{i}\right\}\right): \sum_{n=1}^{\infty}-m_{n}(k)<\infty \Rightarrow \mu$ is absolutely continuous. Thus we have identified a "borderline" between absolute continuity and singular continuity. (By taking $\sum_{n=1}^{\infty}-m_{n}(k)<\infty$ and $\sum_{n=1}^{\infty}-m_{n}(k)=\infty$ respectively for $k$ in disjoint subsets of $[\alpha, \beta]$ we can arrive at a more general class of measures which may include both a singular and an absolutely continuous part.) 
Some of the various possibilities which may occur are illustrated in the following example, for which the $f_{i}(k, y)$ are taken not to be explicitly dependent on $k$ :

Example. Let

$$
\mu_{n}(\Sigma)=\int_{\Sigma} d k \prod_{i=1}^{n}\left(1+g_{i} \sin \left(N_{i} k\right)\right)
$$

and assume $0<g_{i}<1, \lim _{i \rightarrow \infty} g_{i}=0$.

Define $\mu(\Sigma)=\lim _{n \rightarrow \infty} \mu_{n}(\Sigma)$ for subintervals of $[0,1]$, say.

Then, provided the $N_{i}$ increase sufficiently rapidly (in a manner defined more precisely by the proof of Theorem 1 ), it follows that

i) $\sum_{n=1}^{\infty} g_{n}^{2}=\infty \Rightarrow \mu$ is singular continuous.

ii) $\sum_{n=1}^{\infty} g_{n}^{2}<\infty \Rightarrow \mu$ is absolutely continuous. Moreover,

iii) $\sum_{n=1}^{\infty} g_{n}<\infty \Rightarrow \mu$ is absolutely continuous for every sequence $\left\{N_{i}\right\}$, the infinite product

$$
\prod_{i=1}^{\infty}\left(1+g_{i} \sin \left(N_{i} k\right)\right)
$$

then being both absolutely and uniformly convergent.

Theorem 1 may be generalised in various ways for example by weakening the regularity assumptions on the $f_{i}$; however condition iv) is found to hold in most applications to potential scattering. A more useful generalisation, which will be needed in Section 3 is to allow $f_{i}$ to depend explicitly on $\left\{N_{j}\right\}$ for $j<i$. This gives

Corollary to Theorem 1 . Let the functions $f_{n}\left(k ; y ; N_{1} ; N_{2} ; \ldots N_{n-1}\right)$ be periodic in $y$, with period $c$, and for fixed $\left\{N_{i}\right\}$ be continuously differentiable in $k$ and $y$ and satisfy i)-iv) of Theorem 1, where i) holds uniformly in the $\left\{N_{i}\right\}$ (as well as in $k, y$ ) and $m_{n}(k)$ is assumed independent of the $\left\{N_{i}\right\}$. Define a sequence of measures $\mu_{n}$ by

$$
\mu_{n}(\Sigma)=\int_{\Sigma} d k \prod_{i=1}^{n} f_{i}\left(k, N_{i} k ; N_{1}, N_{2}, \ldots, N_{i-1}\right) .
$$

Then the conclusion of Theorem 1 holds.

Proof. Follows closely the proof of the theorem.

3. The Spectral Function of $\frac{-d^{2}}{d r^{2}}+V$ in $L^{2}(0, \infty)$; Examples of Singular

\section{Continuous Spectrum}

We consider first a potential $V(r)$ vanishing except on an infinite sequence of intervals of length $a$, on each of which $V$ is given by some prescribed $L^{1}$ function $W$. 
Let $\left\{N_{i}\right\} \quad(i=1,2,3, \ldots)$ be the separation between consecutive intervals, and suppose that

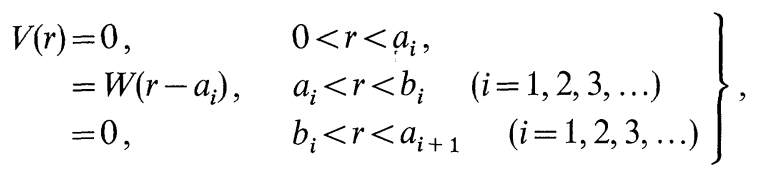

where

$$
\left.\begin{array}{l}
a_{i}=(i-1) a+\sum_{j=1}^{i} N_{j} \\
b_{i}=a+a_{i}=i a+\sum_{j=1}^{i} N_{j}
\end{array}\right\}
$$

Here $W$ and $a$ are given and the sequence $\left\{N_{i}\right\}$ is to be chosen. Setting $W(x) \equiv 0$ for $x \in(0, a)$ we have, then,

$$
V(r)=\sum_{i=1}^{\infty} W\left(r-a_{i}\right)
$$

The spectral function $\varrho(\lambda)$ of the differential operator $-\frac{d^{2}}{d r^{2}}+V(r)$ in $[0, \infty)$, with the boundary condition $\psi(0)=0$, defines a Lebesgue-measure $\mu$ which may be derived from the limit, as $n \rightarrow \infty$, of the measure $v_{n}$ corresponding to the same differential operator in the interval $\left[0, a_{n+1}\right.$ ] [with boundary conditions $\phi(0)=0$ and $\phi\left(a_{n+1}\right)=0$ say].

Restricting attention to subintervals $\Sigma$ of $(0, \infty)$, in which case $\mu$ will be found to be continuous, we have

$$
\mu(\Sigma)=\lim _{n \rightarrow \infty} v_{n}(\Sigma) .
$$

For given $N_{1}, N_{2}, \ldots, N_{n}$, and in the limit $N_{n+1} \rightarrow \infty, v_{n}(\Sigma)$ itself approaches $\mu_{n}(\Sigma)$, the measure derived from the spectral function of $-\frac{d^{2}}{d r^{2}}+V_{n}(r)$ in $[0, \infty)$ with boundary condition $\phi(0)=0$, where

$$
\left.\begin{array}{rlrl}
V_{n}(r) & =V(r), & & 0<r<b_{n} \\
& =0, & & b_{n}<r<\infty
\end{array}\right\} .
$$

Since $\mu_{n}$ is continuous (on $\mathbb{R}^{+}$), for given $N_{1}, N_{2}, \ldots, N_{n}$ the convergence of $v_{n}$ to $\mu_{n}$ is uniform over subintervals $\Sigma$ of any fixed finite interval, and by successively choosing the $N_{n+1}$ sufficiently large such that

$$
\left|v_{n}(\Sigma)-\mu_{n}(\Sigma)\right|<\frac{1}{n}
$$

for subintervals of $\left[n^{-1}, n\right]$ we can ensure that (13) implies

$$
\mu(\Sigma)=\lim _{n \rightarrow \infty} \mu_{n}(\Sigma)
$$

for finite subintervals of $\mathbb{R}^{+}$not having the origin as endpoint. 
For $k>0$, let $\phi(r ; k)$ be the solution of

$$
-\phi^{\prime \prime}(r ; k)+V(r) \phi(r ; k)=k^{2} \phi(r ; k)
$$

subject to the initial conditions

$$
\left.\begin{array}{l}
\phi(0 ; k)=0 \\
\phi^{\prime}(0 ; k)=1
\end{array}\right\}
$$

and define $R(r ; k)$ and $\theta(r ; k)$ by

$$
\left.\begin{array}{l}
\phi=k^{-1} R \cos \theta \\
\phi=R \sin \theta
\end{array}\right\}
$$

with $\theta=\pi / 2$ when $r=0$.

More exactly, $\theta=\tan ^{-1}\left(\phi^{\prime} / k \phi\right)$ is determined by the equation

$$
\frac{d \theta}{d r}=-k+k V \phi^{2} / R^{2}
$$

and we can write

$$
\theta(r)=(\pi / 2)-k r+\int_{0}^{r}\left(k V \phi^{2} / R^{2}\right) d r
$$

We shall have occasion to use the result $\frac{d \theta}{d r}=-k$ wherever $V(r)=0 . R(r)$ is most easily determined from the equation $R^{2}=\left(\phi^{\prime}\right)^{2}+k^{2} \phi^{2}$.

For $\lambda>0$ we set $\lambda=k^{2}$ and

$$
\left.\begin{array}{rl}
\theta_{n}(k) & =\theta\left(b_{n} ; k\right) \\
R_{n}(k) & =R\left(b_{n} ; k\right)
\end{array}\right\} .
$$

The spectral function $\varrho_{n}(k)$ associated with the absolutely continuous measure $\mu_{n}$ is determined by the asymptotic behaviour, as $r \rightarrow \infty$, of solutions $\psi(r ; k)$ of the equation.

$$
-\psi^{\prime \prime}(r ; k)+V_{n}(r) \psi(r ; k)=k^{2}(r ; k) .
$$

With the initial conditions $\psi(0 ; k)=0, \psi^{\prime}(0 ; k)=1,(14)$ implies

$$
\begin{aligned}
\psi\left(b_{n} ; k\right) & =\phi\left(b_{n} ; k\right), \\
\psi^{\prime}\left(b_{n} ; k\right) & =\phi^{\prime}\left(b_{n} ; k\right) .
\end{aligned}
$$

Moreover, $\left(\psi^{\prime}\right)^{2}+k^{2} \psi^{2}=$ const for $r \geqq b_{n}$, and using (18) and (19) we have the asymptotic behaviour

$$
\psi(r ; k)=\frac{R_{n}(k)}{k} \sin \left(k r+\delta_{n}(k)\right)+o(1)
$$

as $r \rightarrow \infty$, from which may be deduced

$$
\frac{d \varrho_{n}(k)}{d k}=\frac{2 k^{2}}{\pi\left[R_{n}(k)\right]^{2}}
$$


$\left(\delta_{n}\right.$ is determined by the equation

$$
\left.k b_{n}+\delta_{n}=\theta_{n}(\bmod 2 \pi)\right) .
$$

Hence,

$$
\mu_{n}(\Sigma)=\int_{\Sigma} \frac{2 k^{2}}{\pi\left[R_{n}(k)\right]^{2}} d k
$$

We shall evaluate the r.h.s. by relating $R_{n}$ to $R_{n-1}$. Consider further the solution $\theta$ of (16) and (17).

Now $\frac{d \theta}{d r}=-k,\left(b_{n-1}<r<b_{n}\right)$, so that

$\theta\left(a_{n} ; k\right)=\theta\left(b_{n-1} ; k\right)-N_{n} \cdot k=\theta_{n-1}(k)-N_{n} k$.

Again $\frac{d R}{d r}=0,\left(b_{n-1}<r<b_{n}\right)$, so that

$$
R\left(a_{n} ; k\right)=R\left(b_{n-1} ; k\right)=R_{n-1}(k) \text {. }
$$

We have then,

$$
\begin{aligned}
& \phi\left(a_{n} ; k\right)=k^{-1} R_{n-1}(k) \cos \left(\theta_{n-1}(k)-N_{n} k\right) \\
& \phi\left(a_{n} ; k\right)=R_{n-1}(k) \sin \left(\theta_{n-1}(k)-N_{n} k\right) .
\end{aligned}
$$

Now define the matrix $M(k)$ by

$$
\left(\begin{array}{l}
u(a) \\
u^{\prime}(a)
\end{array}\right)=M(k)\left(\begin{array}{l}
u(0) \\
u^{\prime}(0)
\end{array}\right)
$$

for solutions of $-u^{\prime \prime}(r)+W(r) u(r)=k^{2} u(r) \quad(0 \leqq r \leqq a)$.

Then,

$$
\left(\begin{array}{c}
\phi\left(b_{n} ; k\right) \\
\phi^{\prime}\left(b_{n} ; k\right)
\end{array}\right)=M(k)\left(\begin{array}{c}
\phi\left(a_{n} ; k\right) \\
\phi^{\prime}\left(a_{n} ; k\right)
\end{array}\right)
$$

Using (23) and (24) to evaluate $\phi$ and $\phi^{\prime}$ at $r=b_{n}$ and noting that $R_{n}(k)=\left[\phi^{\prime}\left(b_{n} ; k\right)\right]^{2}+k^{2}\left[\phi\left(b_{n} ; k\right)\right]^{2}$ we find

$$
\begin{aligned}
\left(\frac{R_{n}(k)}{R_{n-1}(k)}\right)^{2}= & {\left[\left(M_{11}(k)\right)^{2}+k^{-2}\left(M_{21}(k)\right)^{2}\right] \cos ^{2}\left(\theta_{n-1}(k)-N_{n} k\right) } \\
& +\left[\left(M_{22}(k)\right)^{2}+k^{2}\left(M_{12}(k)\right)^{2}\right] \sin ^{2}\left(\theta_{n-1}(k)-N_{n} k\right) \\
& +2\left[k M_{11}(k) M_{12}(k)+k^{-1} M_{21}(k) M_{22}(k)\right] \\
& \cdot \sin \left(\theta_{n-1}(k)-N_{n} k\right) \cos \left(\theta_{n-1}(k)-N_{n} k\right) .
\end{aligned}
$$

We shall denote by $f_{n}\left(k, N_{n} k ; \theta_{n-1}(k)\right)$ the inverse of the r.h.s., so that $\left(\frac{R_{n}(k)}{R_{n-1}(k)}\right)^{2}=\left(f_{n}\left(k, N_{n} k, \theta_{n-1}(k)\right)\right)^{-1}$ 
with

$$
f_{n}(k, y, \theta)^{-1}=A(k)+B(k) \cos 2(\theta-y)+C(k) \sin 2(\theta-y),
$$

where

$$
\begin{aligned}
& A=\frac{1}{2}\left(M_{11}^{2}+M_{22}^{2}+k^{-2} M_{21}^{2}+k^{2} M_{12}^{2}\right) \\
& B=\frac{1}{2}\left(M_{11}^{2}+k^{-2} M_{21}^{2}-M_{22}^{2}-k^{2} M_{12}^{2}\right) \\
& C=\left(k M_{11} M_{12}-k^{-1} M_{21} M_{22}\right) .
\end{aligned}
$$

These coefficients are not independent, since we find

$$
A^{2}-B^{2}-C^{2}=(\operatorname{det} M)^{2}=1 .
$$

Using the formulae

$$
\int_{0}^{2 \pi} \frac{d z}{a+b \cos z}=\frac{2 \pi}{\sqrt{a^{2}-b^{2}}}
$$

and

$$
\int_{0}^{2 \pi} d z \log (a+b \cos z)=2 \pi \log \left(\frac{a+\sqrt{a^{2}-b^{2}}}{2}\right),
$$

we find

$$
\overline{f_{n}} \equiv \frac{1}{\pi} \int_{0}^{\pi} f_{n}(k, y, \theta) d y=\frac{1}{\sqrt{A^{2}-B^{2}-C^{2}}}=1
$$

and

$$
m_{n}(k) \equiv \frac{1}{\pi} \int_{0}^{\pi} \log f_{n}(k, y, \theta) d y=\log \left(\frac{2}{A+1}\right) .
$$

Using (22) and (25) we have

$$
\mu_{n}(\Sigma)=\int_{\Sigma} d k \frac{2 k^{2}}{\pi} \prod_{i=1}^{n} f_{i}\left(k, N_{i} k, \theta_{i-1}(k)\right),
$$

where $f_{i}$, through $\theta_{i-1}$, depends on $N_{1}, N_{2}, \ldots, N_{i-1}$.

We first note that $m_{n}(k)$, which in fact in this case is independent of $n$, cannot vanish identically.

\section{Proof.}

$m(k) \equiv 0 \Rightarrow A(k) \equiv 1 \Rightarrow B(k)=C(k) \equiv 1 \Rightarrow f_{n} \equiv 1$.

We have, then, $R_{n}(k)=R_{n-1}(k)$, so that (22) implies

$\varrho_{n}(k)=\varrho_{n-1}(k), \quad\left(\lambda=k^{2}>0\right)$.

By analytical continuation to $\lambda=-k^{2} \leqq 0$, the solution $\psi$ of $-\psi^{\prime \prime}+V_{n} \psi=-k^{2} \psi$ 
with initial conditions $\psi(0)=0, \psi^{\prime}(0)=1$, satisfies $\left(\psi^{\prime}\left(b_{n}\right)\right)^{2}-k^{2}\left(\psi\left(b_{n}\right)\right)^{2}=1$. But for $\lambda=-k^{2}$ to be an eigenvalue of $-\frac{d^{2}}{d r^{2}}+V_{n}$ would require $\left(\psi^{\prime}\left(b_{n}\right)\right)^{2}-k^{2}\left(\psi\left(b_{n}\right)\right)^{2}=0$. Hence $-\frac{d^{2}}{d r^{2}}+V_{n}$ has no eigenvalues $\lambda \leqq 0$, and $d \varrho_{n}=d \varrho_{n-1}=0$ for $\lambda<0$.

It follows that $\varrho_{n}=\varrho_{n-1}$. But the potential is uniquely determined by the spectral function, and we must have $V_{n} \equiv V_{n-1}$, which is only satisfied when $V \equiv 0$.

Since $m(k)$ is analytic, it follows that $m(k)=0$ at only isolated points which, it may be verified, do not belong to the point spectrum of $-\frac{d^{2}}{d r^{2}}+V$. The corollary to Theorem 1 may now be applied. [Note that i):

$(28) \Rightarrow A \geqq 1 \Rightarrow m(k) \leqq 0 \Rightarrow \sum_{n=1}^{\infty} m(k)=-\infty$

except where $m(k)=0$.

ii): Proofs are unaffected by the factor $2 k^{2} / \pi$ on the r.h.s. of (30).

We have, then,

Proposition 1. Let $V(r)$ be defined by (11) and (12). Then, provided the $\left\{N_{i}\right\}$ increase sufficiently rapidly, the differential operator $-\frac{d^{2}}{d r^{2}}+V$ in $[0, \infty)$ has singular continuous spectrum for $\lambda>0$.

Equations (11) and (12) define, then, a simple class of potentials with which we are able to associate a singular continuous spectrum. The potential consists of an infinite sequence of "bumps" of identical (but arbitrary) shape, with separation increasing rapidly with distance. This is the basic idea of what in Section 4 we shall set in a more general context. Here let us note that a class of potentials for which $\lim _{r \rightarrow \infty} V(r)=0$ is obtained by decreasing the height of bumps with distance.

Let

$$
\begin{aligned}
V(r) & =0 & & 0<r<a_{1} \\
& =g_{i} W\left(r-a_{i}\right), & & a_{i}<r<b_{i} \\
& =0, & & b_{i}<r<a_{i+1},
\end{aligned}
$$

where the sequences $\left\{a_{i}\right\},\left\{b_{i}\right\}$ are again given by (12), and assume $\lim _{n \rightarrow \infty} g_{n}=0$.

The treatment of this case is essentially as before, except that the transfer matrices $M$ at the $n$ 'th bump, and the coefficients $A, B, C$, now depend on $n$. From the perturbation series for $A_{n}$, we have

$$
A_{n}=1+\frac{g_{n}^{2}}{2 k^{2}}\left(\left(\int_{0}^{c} d x W(x) \cos 2 k x\right)^{2}+\left(\int_{0}^{c} d x W(x) \sin 2 k x\right)^{2}\right)+0\left(g_{n}^{3}\right) .
$$

According to (29), this gives

$$
m_{n}(k) \sim-\frac{g_{n}^{2}}{4 k^{2}}\left(\left(\int_{0}^{c} d x W(x) \cos 2 k x\right)^{2}+\left(\int_{0}^{c} d x W(x) \sin 2 k x\right)^{2}\right) .
$$


From the Corollary to Theorem 1 , the nature of the measure $\mu$ depends now on the convergence or divergence of $\sum_{n=1}^{\infty} g_{n}^{2}$, and we have

Proposition 2. Let $V(r)$ be given by (31), with $\lim _{n \rightarrow \infty} g_{n}=0$. Then for $\lambda>0$ the differential operator $-\frac{d^{2}}{d r^{2}}+V$ in $[0, \infty)$ has singular continuous spectrum if $\sum_{n=1}^{\infty} g_{n}^{2}<\infty$

\section{Interpretation of Singular Continuous Spectrum}

From Equation (29), one finds that $m_{n}(k)$ is exactly the logarithm of the transition probability of a particle through a single bump, in the absence of other contributions to the potential. The condition $\sum_{n=1}^{\infty}-m_{n}=\infty$ thus corresponds to the vanishing of the infinite product of successive transition probabilities and expresses the certainty that the particle will ultimately be reflected from a bump.

For the classical probabilistic stochastic process of a particle meeting an infinite sequence of barriers, let $p_{n}$ be the probability of transmission and $q_{n}$ of reflection $\left(p_{n}+q_{n}=1\right)$. The process is said to be recurrent if the particle returns to the origin, with probability 1 , on infinitely many occasions, and transient otherwise. (In both cases, unless some $p_{n}=0$, the particle will ultimately be transmitted at least once through each barrier.) The condition for recurrence is found to be $\sum_{n=1}^{\infty}\left(q_{n} / p_{n}\right)=\infty$, which corresponds exactly, for the potentials defined by (31), to the condition $\sum_{n=1}^{\infty} g_{n}^{2}=\infty$. That is, one has singular continuous spectrum if and only if the classical process is recurrent. The physical picture is then of a particle performing a kind of (quantum!) random walk, in which however great the distance from the origin, the effect on the particle of the potential at finite distances cannot be ignored. (This classical analogy becomes appropriate in the limiting case as the $\left\{N_{i}\right\}$ increase arbitrarily rapidly, since in the limit successive transition probabilities are "independent".)

Let us examine more closely the quantum mechanical aspects of this phenomenon. Let $V_{\text {s.c. }}(r)$ be a potential giving rise to purely singular continuous spectrum for the differential operator $-\frac{d^{2}}{d r^{2}}+V_{\text {s.c. }}(r)$ in $L^{2}(0, \infty)$; (the argument may be generalised to allow a discrete or absolutely continuous component) and let

$$
H=-\frac{d^{2}}{d x^{2}}+V_{0}(x)
$$

in $L^{2}(\mathbb{R})$, where

$$
\begin{aligned}
V_{0}(x) & =V_{\text {s.c. }}(x), & & x>0 \\
& =0 & & x<0 .
\end{aligned}
$$


We may also define the self-adjoint operator $\tilde{H}=-\frac{d^{2}}{d x^{2}}+V_{0}(x)$ acting in $L^{2}(\mathbb{R})$ $=L^{2}\left(\mathbb{R}^{+}\right)+L^{2}\left(\mathbb{R}^{-}\right)$with the two regions "decoupled". [I.e. with boundary conditions $f(0+)=f(0-)=0$; here

$$
\left.L^{2}\left(\mathbb{R}^{ \pm}\right) \equiv\left\{g \in L^{2}(\mathbb{R}) ; g(x)=0 \quad \text { for } \quad x \lessgtr 0\right\} .\right]
$$

Then standard arguments based on trace conditions ([4], p. 649) show that

i) $\operatorname{slim}_{t \rightarrow \pm \infty} e^{i \tilde{H} t} e^{-i H t}$ exists on $\mathrm{M}_{\text {a.c. }}(H)$ and has range in $M_{\text {a.c. }}(\tilde{H}) \equiv L^{2}\left(\mathbb{R}^{-}\right)$;

ii) $\underset{t \rightarrow \pm \infty}{S-\lim _{t \rightarrow \infty}} e^{i H t} e^{-i \tilde{H} t}$ exists on $M_{\text {a.c. }}(\tilde{H})$;

iii) $\underset{t \rightarrow \pm \infty}{S-\lim _{t \rightarrow \infty}} e^{i H t} E_{x<0} e^{-i H_{0} t}$ exists on $\mathscr{H}$, where $H_{0}=-\frac{d^{2}}{d x^{2}}$ in $L^{2}(\mathbb{R})$ and $E_{x<0}$ is the projection onto $L^{2}\left(\mathbb{R}^{-}\right)$; moreover

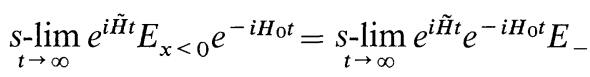

and

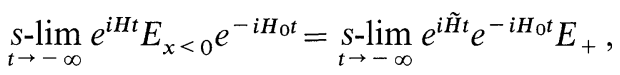

where $E_{ \pm}$are the respective projections onto subspaces $M_{ \pm}$of positive and negative momentum.

Transitivity now implies the existence, on $M_{+}$and $M_{-}$respectively, of the wave operators $\Omega_{+}\left(H, H_{0}\right)=\underset{t \rightarrow-\infty}{s-\lim _{t}} e^{i H t} e^{-i H_{0} t}$ and

$$
\Omega_{-}\left(H, H_{0}\right)=\underset{t \rightarrow+\infty}{S-\lim _{t \rightarrow \infty}} e^{i H t} e^{-i H_{0} t} .
$$

These wave operators are complete; i.e. range $\left(\Omega_{+}\right)=$range $\left(\Omega_{+}\right)=M_{\text {a.c. }}(H)$. An incoming particle from $x=-\infty$ will be reflected with probability 1 . Thus $V_{\text {s.c. }}(r)$ represents a totally reflecting barrier. We can confirm this interpretation in the case $H=-\frac{d^{2}}{d r^{2}}+V(r)$, acting in $L^{2}(0, \infty)$, with $V$ given by (11).

Using (26) and (30) with the method of Section 2 we find

$\lim _{n \rightarrow \infty} \int e^{-i k x_{n}} d \mu_{n} \neq 0 \quad\left(x_{n}=2 N_{n}\right)$.

(This uses the fact that $\int_{0}^{2 \pi} \frac{d z e^{-i z}}{a+b \cos z} \neq 0$.)

If the $\left\{N_{n}\right\}$ increases sufficiently rapidly, this implies that

$$
\lim _{n \rightarrow \infty} \int e^{-i k x_{n}} d \mu \neq 0 \text {. }
$$

A similar result holds for each of the sequences $x_{n}=2 c N_{n}(c= \pm 2, \pm 3, \ldots)$, and for integer linear combinations, such as $x_{n}=2\left(N_{n}+N_{n+1}\right)$. So the spectral measure $\mu$ for the differential operator $H=\frac{-d^{2}}{d r^{2}}+V$ in this case does not obey the RiemannLebesgue Lemma.

Consider now a normalised wave packet $f$ having a narrow range $\delta E$ of total energy [for the time intervals in which we are interested we shall suppose 
$(\delta k)^{2} t \ll 1$, where $\delta E=\delta\left(k^{2}\right)=2 k_{0} \delta k$; this ensures that the wave packet does not spread too much. But we can allow $(\delta E), t \gg 1]$.

We have, for some $p \in L^{2}\left(\mathbb{R}^{+}, d \mu\right)$,

$$
\left\langle f, e^{-i H t} f\right\rangle=\int p(k) e^{-i k^{2} t} d \mu .
$$

Making the change of variables $k=k_{0}+q$ and neglecting terms of order $q^{2} t$ we find that $\left\langle f, e^{-i H t} f\right\rangle$ is appreciably different from zero for the sequence of times $t=t_{n}$ given by $k_{0} t_{n}=N_{n}$. Each instant of this sequence corresponds physically to the time taken for the wave packet to return to its original position and direction of motion after a single reflection at the $n$ 'th bump and at $r=0$. (Note that we have units in which $m=\frac{1}{2}$, so that with $k_{0}=m v_{0}$ we have $v_{0} t_{n}=2 N_{n}$.) Moreover, $t= \pm c t_{n}$ corresponds to multiple reflections at the $n^{\prime}$ th bump, and integer linear combinations relate to the possibility of successive reflections at a number of different bumps. This is confirmed by a detailed analysis, using the appropriate generalised Fourier transforms, of the development of a wave packet in position space. The method of stationary phase exhibits, for example, components of the wave packet centred around

$$
r=r_{0}-2 c N_{n}+2 k_{0} t \text {. }
$$

Since it is known ([2]) that the mean squared probability, for $f \in M_{c}(H)$, of finding the particle in any fixed bounded region, converges to zero, the wave packet will ultimately be at a great distance from $r=0$ "for most of the time", but will always return for a limited interval of time, to a neighbourhood of the origin. A similar interpretation holds in the case of the potential $V$ defined by (31).

\section{General Framework for Singular Continuous Spectrum in Potential Scattering}

The arguments of Section 3, relating the nature of the spectrum of the Hamiltonian to asymptotic behaviour of solutions $\phi$ of the time-independent Schrödinger equation, make essential use of the behaviour of $\phi$ only at discrete sets of points. Instead of having to discuss the manner in which $\phi$ varies continuously with $r$, one determines $\phi$ at discrete sequences of points $\left\{a_{i}\right\}$ and $\left\{b_{i}\right\}$, and estimates the transfer matrices relating $\phi$ and $\phi^{\prime}$ at points of these sequences. We now proceed to generalise this approach in terms of transfer matrices to deal with a larger class of potentials, including in particular singular short range potentials for which the Hamiltonian has singular continuous spectrum.

Let $\left\{a_{i}\right\}$ and $\left\{b_{i}\right\}\left(b_{i}>a_{i} ; i=1,2,3, \ldots\right)$ be two such (increasing) sequences and to each value of $i$ associate a further increasing sequence $\left\{x_{j}^{(i)}\right\}(j=0,1,2, \ldots)$ satisfying

$$
x_{0}^{(i)}=b_{i-1} \quad \text { and } \quad \lim _{j \rightarrow \infty} x_{j}^{(i)}=x_{\infty}^{(i)}, \quad \text { say . }
$$

Let $a_{i}=x_{N_{i}}^{(i)}$, so that the sequence $\left\{x_{j}^{(i)}\right\}\left(j=0,1,2, \ldots, N_{i}\right)$ defines a partition of $\left(b_{i-1}, a_{i}\right)$ into $N_{i}$ subintervals. [Equation (12) corresponds to the case $x_{j+1}^{(i)}=x_{j}^{(i)}+1$ and $b_{i}=a_{i}+a$, but other sequences may be appropriate, depending on the class of potentials. As in Section 3 , the $N_{i}$ 's are taken to be rapidly increasing with $i$.] 
The potential $V(r)$ is to be given in terms of sequences $\left\{V^{(i)}(r)\right\}$ and $\left\{W^{(i)}(r)\right\}$ $(i=1,2,3, \ldots)$ of real locally $L^{1}$ functions, defined respectively on the intervals

$$
b_{i-1}=x_{0}^{(i)}<r<x_{\infty}^{(i)}
$$

and

$$
a_{i}<r<b_{i} .
$$

We take, then,

$$
\begin{aligned}
& V(r)=V^{(1)}(r) \quad b_{0}=0<r<a_{1} \\
& =W^{(i)}(r) \quad a_{i}<r<b_{i} \quad(i=1,2,3, \ldots) \\
& =V^{(i)}(r) \quad x_{0}^{(i)}=b_{i-1}<r<a_{i}=x_{N_{\imath}}^{(i)} \quad(i=1,2,3, \ldots)
\end{aligned}
$$

Each $N_{i}$ thus determines the distance in position space over which the potential is given by $V^{(i)}(r)$. [Equation (11) corresponds to the case $V^{(i)}(r) \equiv 0$, $W^{(i)}(r)=W\left(r-a_{i}\right)$. In applications there will be some algorithm for inductively specifying $V^{(n)}(r)$ and the sequence $\left\{x_{j}^{(n)}\right\}$ once the potential is known in the interval $0<r<b_{n-1}$.]

Let us denote by $M_{n}(\lambda)$ the $(2 \times 2)$ transfer matrix satisfying

$$
M_{n}\left(\begin{array}{c}
\psi\left(a_{n}\right) \\
\psi^{\prime}\left(a_{n}\right)
\end{array}\right)=\left(\begin{array}{c}
\psi\left(b_{n}\right) \\
\psi^{\prime}\left(b_{n}\right)
\end{array}\right)
$$

for solutions $\psi(r)$ of $-\psi^{\prime \prime}+W^{(n)} \psi=\lambda \psi$, and by $t_{j, n}(\lambda)$ the transfer matrix satisfying

$$
t_{j, n}\left(\begin{array}{l}
\psi\left(x_{0}^{(n+1)}\right) \\
\psi^{\prime}\left(x_{0}^{(n+1)}\right)
\end{array}\right)=\left(\begin{array}{l}
\psi\left(x_{j}^{(n+1)}\right) \\
\psi^{\prime}\left(x_{j}^{(n+1)}\right)
\end{array}\right)
$$

for solutions $\psi(r)$ of

$$
-\psi^{\prime \prime}+V^{(n+1)} \psi=\lambda \psi .
$$

"Discrete wave operators" may be defined for each $n$, which describe the asymptotic behaviour of $\left(\begin{array}{l}\psi\left(x_{j}^{(n+1)}\right) \\ \psi^{\prime}\left(x_{j}^{(n+1)}\right)\end{array}\right)$ in the limit as $j \rightarrow \infty$. We suppose that there exists some real matrix $t(\lambda)$, continuously differentiable with respect to $\lambda$ in some closed subinterval of $\mathbb{R}$, and having complex eigenvalues $\exp ( \pm i \beta(\lambda))(\beta$ real $)$, for which the following uniform limits exist:

$$
\left.\begin{array}{rl}
w^{(n)}(\lambda) & =\lim _{j \rightarrow \infty}\left(t^{(n)}\right)^{-j} t_{j, n} \\
\frac{d}{d \lambda} w^{(n)}(\lambda) & =\lim _{j \rightarrow \infty} \frac{d}{d \lambda}\left[\left(t^{(n)}\right)^{-j} t_{j, n}\right]
\end{array}\right\} .
$$

Let $f_{ \pm}$be eigenvectors of $t$ corresponding to eigenvalues $\exp ( \pm i \beta)$ respectively, and chosen to satisfy $f_{-}=\left(f_{+}\right)^{*}$. Denoting by $f_{ \pm i}(i=1,2)$ the $i$ th component of $f_{ \pm}, f_{+1} f_{-2}-f_{+2} f_{-1}$ is pure imaginary, so that the $f_{ \pm}$may be "normalised" to satisfy

$$
f_{+1} f_{-2}-f_{+2} f_{1}=-i \frac{d \beta}{d \lambda} .
$$


(A priori we could write only $\pm i \frac{d \beta}{d \lambda}$, but we shall subsequently confirm the sign of the r.h.s.)

We may now use the discrete wave operators $w^{(n)}$ to define, for real solutions $\phi$ of $-\phi^{\prime \prime}+V \phi=\lambda \phi$, discrete analogues of the $R$ and $\theta$ variables of Section 3 .

First write

$$
w^{(n)}\left(\begin{array}{l}
\phi\left(b_{n}\right) \\
\phi^{\prime}\left(b_{n}\right)
\end{array}\right)=p_{n} f_{+}+q_{n} f_{-} \quad\left(p_{n}=q_{n}^{*}\right),
$$

and then define real $R_{n}$ and $\theta_{n}(\bmod 2 \pi)$ by

$$
p_{n}=i R_{n} e^{i \theta_{n}}, \quad q_{n}=-i R_{n} e^{-i \theta_{n}}
$$

with $R_{n}=+\sqrt{\left|p_{n}\right|^{2}+\left|q_{n}\right|^{2}}$.

As in Section 3 we shall assume that the $N_{i}$ increase sufficiently rapidly that the spectral measure of $-\frac{d^{2}}{d r^{2}}+V$ (in the interval $[0, b)$, where $\left.b=\lim _{n \rightarrow \infty} b_{n}\right)$ may be derived by a limiting argument as $n \rightarrow \infty$ from the spectral function $\varrho_{n}$ of the differential operator $-\frac{d^{2}}{d r^{2}}+V_{n}\left(\right.$ in the interval $\left.\left[0, x_{\infty}^{(n)}\right)\right)$ where

$$
\begin{aligned}
V_{n}(r) & =V(r) & & 0<r<b_{n} \\
& =V^{(n)}(r) & & b_{n}<r<x_{\infty}^{(n)} .
\end{aligned}
$$

[We take always the boundary condition of $\psi(O)=0$.] For the solution $\psi$ of $-\psi^{\prime \prime}$ $+V_{n} \psi=\lambda \psi$ with initial conditions $\psi(O)=0, \psi^{\prime}(O)=1,(40)$ implies

$$
\left(\begin{array}{l}
\psi\left(x_{j}^{(n)}\right) \\
\psi^{\prime}\left(x_{n}^{(n)}\right)
\end{array}\right)=p_{n} e^{i n \beta} f_{+}+q_{n} e^{-i n \beta} f_{-}+o(1)
$$

as $n \rightarrow \infty$, where $p_{n}$ and $q_{n}$ satisfy (42) with $\phi\left(b_{n}\right)=\psi\left(b_{n}\right)$ and $\phi^{\prime}\left(b_{n}\right)=\psi^{\prime}\left(b_{n}\right)$.

Employing the notation $\left\{\begin{array}{l}a(\lambda) \\ b(\lambda)\end{array}\right\}=b \frac{d a}{d \lambda}-a \frac{d b}{d \lambda}$, we have

$$
\int_{0}^{x_{j}^{(n)}}(\psi(r))^{2} d r=\left\{\begin{array}{l}
\psi\left(x_{j}^{(n)}\right) \\
\psi^{\prime}\left(x_{j}^{(n)}\right)
\end{array}\right\}
$$

Using the estimates for $\psi$ and $\psi^{\prime}$, and for derivatives with respect to $\lambda$, this gives

$$
\begin{aligned}
\int_{0}^{x_{j}^{(n)}}(\psi(r))^{2} d r & =2 \operatorname{in} \frac{d \beta}{d \lambda} p_{n} q_{n}\left(f_{+1} f_{-2}-f_{+2} f_{-1}\right)+o(1) \\
& =2 n\left(R_{n} \frac{d \beta}{d \lambda}\right)^{2}+o(1), \quad \text { using (41) and (43). }
\end{aligned}
$$

[This justifies the sign of the r.h.s. of (41).] 
We can also estimate the distribution of eigenvalues of $-\frac{d^{2}}{d r^{2}}+V_{n}$ acting in $L^{2}\left(0, x_{j}^{(n)}\right)$ and deduce that

$$
\frac{d \varrho_{n}}{d \beta}=\left[2 \pi\left(R_{n} \frac{d \beta}{d \lambda}\right)^{2}\right]^{-1} \text {. }
$$

Using $\beta$ to parametrise the points of a subset $\Sigma$ of $\mathbb{R}$, this gives for the measure $\mu_{n}$ corresponding to $\varrho_{n}$

$$
\mu_{n}(\Sigma)=\int_{\Sigma}\left[2 \pi\left(R_{n} \frac{d \beta}{d \lambda}\right)^{2}\right]^{-1} d \beta,
$$

where (21) and (22) correspond to the case $\beta(\lambda)=\lambda^{1 / 2}=k$. Again, we have

$$
\mu(\Sigma)=\lim _{n \rightarrow \infty} \mu_{n}(\Sigma)
$$

for subintervals $\Sigma$.

Thus again $R_{n}$ plays the role of determining the measure $\mu_{n}$, and to complete the analogy with Section 3 we need only evaluate $R_{n} / R_{n-1}$. Now for solutions $\phi$ of $-\phi^{\prime \prime}+V \phi=\lambda \phi$ we have

$$
\left(\begin{array}{l}
\phi\left(a_{n}\right) \\
\phi^{\prime}\left(a_{n}\right)
\end{array}\right)=\tilde{p}_{n-1} e^{i N_{n} \beta} f_{+}+\tilde{q}_{n-1} e^{-i N_{n} \beta} f_{-},
$$

where as $N_{n}$ is taken arbitrarily large, $\tilde{p}_{n-1} \rightarrow p_{n-1}$ and $\tilde{q}_{n-1} \rightarrow q_{n-1}$.

Hence

$$
\left(\begin{array}{l}
\phi\left(b_{n}\right) \\
\phi^{\prime}\left(b_{n}\right)
\end{array}\right)=\tilde{p}_{n-1} e^{i N_{n} \beta} M_{n} f_{+}+\tilde{q}_{n-1} e^{-i N_{n} \beta} M_{n} f_{-} .
$$

Let us write

$$
\left.\begin{array}{l}
w^{(n)} M_{n} f_{+}=U_{11}^{(n)} f_{+}+U_{12}^{(n)} f_{-} \\
w^{(n)} M_{n} f_{-}=U_{21}^{(n)} f_{+}+U_{22}^{(n)} f_{-}
\end{array}\right\} .
$$

Since $\operatorname{det} w^{(n)} M_{n}=1$, this matrix "conserves" $f_{+1} f_{-2}-f_{+2} f_{-1}$, so that again $\operatorname{det} U^{(n)}=1$.

We have, then,

$$
\begin{aligned}
w^{(n)}\left(\begin{array}{l}
\phi\left(b_{n}\right) \\
\phi^{\prime}\left(b_{n}\right)
\end{array}\right)= & \left(U_{11}^{(n)} \tilde{p}_{n-1} e^{i N_{n} \beta}+U_{21}^{(n)} \tilde{q}_{n-1} e^{-i N_{n} \beta}\right) f_{+} \\
& +\left(U_{12}^{(n)} \tilde{p}_{n-1} e^{i N_{n} \beta}+U_{22}^{(n)} \tilde{q}_{n-1} e^{-i N_{n} \beta}\right) f_{-} \\
= & p_{n} f_{+}+q_{n} f_{-} .
\end{aligned}
$$

Using (43) and writing $\tilde{p}_{n-1}=-i \tilde{R}_{n-1} e^{i \tilde{\theta}_{n-1}}$,

$$
\tilde{q}_{n-1}=i \tilde{R}_{n-1} e^{-i \tilde{\theta}_{n-1}},
$$

we find

$$
\left(\frac{R_{n}}{\tilde{R}_{n-1}}\right)^{2}=\left[f_{n}\left(\beta, N_{n} \beta ; \tilde{\theta}_{n-1}(k)\right)\right]^{-1},
$$


where

$$
\left[f_{n}(\beta, y ; \theta)\right]^{-1}=A_{n}+B_{n} \cos 2(y+\theta)+C_{n} \sin 2(y+\theta)
$$

and

$$
\left.\begin{array}{l}
A_{n}=\left(U_{11}^{(n)} U_{22}^{(n)}+U_{21}^{(n)} U_{12}^{(n)}\right) \\
B_{n}=-\left(U_{11}^{(n)} U_{12}^{(n)}+U_{21}^{(n)} U_{22}^{(n)}\right) \\
C_{n}=-i\left(U_{11}^{(n)} U_{12}^{(n)}-U_{21}^{(n)} U_{22}^{(n)}\right.
\end{array}\right\} .
$$

Since $\tilde{R}_{n-1} \rightarrow R_{n-1}, \tilde{\theta}_{n-1} \rightarrow \theta_{n-1}$ as each $N_{n}$ is taken arbitrarily large (holding $N_{i}$ fixed for $i<n$ ), we shall assume that the sequence $\left\{N_{n}\right\}$ has been chosen to increase sufficiently rapidly that the infinite products

$$
\prod_{i=1}^{\infty}\left(R_{i} / \tilde{R}_{i}\right) \text { and } \prod_{i=1}^{\infty} f_{i}\left(\beta, N_{i} \beta, \tilde{\theta}_{i-1}\right) / f_{i}\left(\beta, N_{i} \beta, \theta_{i-1}\right)
$$

converge uniformly to limits which are bounded above and below by strictly positive constants.

In that case, we may define a new family $\left\{\tilde{\mu}_{n}\right\}$ of measures and a limiting measure $\tilde{\mu}$ by

$$
\tilde{\mu}_{n}(\Sigma)=\int_{\Sigma} d \beta\left[2 \pi\left(\frac{d \beta}{d \lambda}\right)^{2}\right]^{-1} \prod_{i=1}^{n} f_{i}\left(\beta, N_{i} \beta, \theta_{i-1}\right),
$$

$\tilde{\mu}(\Sigma)=\lim _{n \rightarrow \infty} \tilde{\mu}_{n}(\Sigma)$ for subintervals $\Sigma$, such that the spectral measure $\mu$ associated with the differential operator $\frac{d^{2}}{d r^{2}}+V$ is equivalent to the measure $\tilde{\mu}$.

In particular, $\mu$ is singular continuous if and only if $\tilde{\mu}$ is singular continuous. We are now in a position to apply the Corollary to Theorem 1 . Noting that $A_{n}$, $B_{n}, C_{n}$ are real, we have

$$
\overline{f_{n}} \equiv \frac{1}{\pi} \int_{0}^{\pi} f_{n}(\beta, y, \theta) d y=\frac{1}{\sqrt{A_{n}^{2}-B_{n}^{2}-C_{n}^{2}}},
$$

where

$$
\begin{aligned}
A_{n}^{2}-B_{n}^{2}-C_{n}^{2}= & \left(U_{11}^{(n)} U_{22}^{(n)}+U_{21}^{(n)} U_{12}^{(n)}\right)^{2} \\
& -4 U_{11}^{(n)} U_{22}^{(n)} U_{21}^{(n)} U_{12}^{(n)} \\
= & \left(\operatorname{det} U^{(n)}\right)^{2}=1 .
\end{aligned}
$$

Hence $\overline{f_{n}}=1$, and we also have

$$
m_{n} \equiv \frac{1}{\pi} \int_{0}^{\pi} \log f_{n}(\beta, y, \theta) d y=\log \left(\frac{2}{A_{n}+1}\right)
$$

so that $m_{n}<0$.

We now have 
Proposition 3. Let $V(r)$ be given by (36) and assume that discrete wave operators $w^{(n)}$ exist, satisfying (40). Define $m_{n}$ as above and suppose that $\sum_{n=1}^{\infty}-m_{n}=\infty$ (uniformly in $\beta$ ). Then, provided the $\left\{N_{i}\right\}$ increase sufficiently rapidly, the differential operator $-\frac{d^{2}}{d r^{2}}+V$ has singular continuous spectrum.

Proposition 3 extends the class of potentials with which we can associate singular continuous spectrum. In any particular case it is necessary to obtain estimates of transfer matrices in terms of which appropriate discrete wave operators can be defined. These estimates can be carried out in the following cases, where "sequence of bumps" means a potential given by (11) and (12) (or more generally by (31) and (12) in the case $\left.\sum_{i=1}^{\infty} g_{i}^{2}=\infty\right)$. It is always assumed that the $\left\{N_{i}\right\}$ increase sufficiently rapidly for Theorem 1 and its corollary to be applied.

Examples. i) Let $V(r)$ be any $L^{1}(o, \infty)$ potential plus a sequence of bumps. Then $-\frac{d^{2}}{d r^{2}}+V+\frac{l(l+1)}{r^{2}}$ has singular continuous spectrum for $\lambda>0$. (I.e. the spectrum is singular continuous for each partial wave.)

ii) Let $V(r)$ be any locally $L^{1}$ periodic potential, plus a sequence of bumps (a periodic lattice with impurities!) Then $-\frac{d^{2}}{d r^{2}}+V$ has singular continuous spectrum (for $\lambda$ in the absolutely continuous spectrum of the unperturbed periodic differential operator).

iii) Let $V(r)=\sum_{i=1}^{\infty} W\left(r-N_{i}\right)$ for $W \in L^{1}(o, \infty)$. Then $-\frac{d^{2}}{d r^{2}}+V$ has singular continuous spectrum for $\lambda>0$.

iv) Add to the short range potential defined in [15] a perturbation $\sum_{i=1}^{\infty} \delta\left(r-x_{N_{i}}\right)$. (For the definition of $\left\{x_{k}\right\}$ in this case see [15]). (Or the $\delta$ 's may be replaced by appropriate locally bounded $\delta$-approximating functions). Then $-\frac{d^{2}}{d r^{2}}+V$ acting in $L^{2}(O, b)$ has singular continuous spectrum in $0<\lambda<\frac{12}{5}$.

v) Let

$$
\begin{aligned}
\tilde{V}(r) & =V(b-r)-1 & & 0<r<b \\
& =0 & & r>b,
\end{aligned}
$$

where $V$ is the potential in iv) above.

Then $-\frac{d^{2}}{d r^{2}}+\tilde{V}$, acting in $L^{2}(O, \infty)$, has singular continuous spectrum for $-1<\lambda<0$.

Here $V$ is short range, but highly singular and oscillating ar $r=0$. One may show (cf. [4]) that the wave operators $\Omega_{ \pm}\left(H_{0}+\tilde{V}, H_{0}\right)$ exist and are complete. 
In each of the preceeding examples i)-iv) a Hamiltonian giving rise to absolutely continuous spectrum is perturbed by a sequence of potential barriers. The effect of the perturbation is to change the nature of the continuous spectrum, making it singular. The perturbation, which may be relatively compact, thus has a profound effect on the spectral properties of the total Hamiltonian. The singular nature of the perturbed spectrum may itself be extremely stable for a wide class of "small" variations of the potential (e.g. change of coupling constant). Perhaps it is absolute continuity that must be regarded as pathological, and singular continuity as the norm!

\section{References}

1. Kato, T.: Perturbation theory for linear operators. Berlin-Heidelberg-New York: Springer 1966

2. Amrein, W., Georgescu, V.: Helv. Phys. Acta 46, 635-658 (1973)

3. Wilcox, C.: J. Funct. Anal. 12, 257-274 (1973)

4. Pearson,D.: Helv. Phys. Acta 48, 639-653 (1975)

5. Reed,M., Simon,B.: Methods of modern mathematical physics, Vol. I. New York-London: Academic Press 1972

6. Weidmann,J.: Math. Zeitschr. 98, 268-302 (1967)

7. Lavine, R.: Indiana Univ. Math. J. 21, 643-656 (1972)

8. Lavine, R.: J. Funct. Anal. 12, 30-54 (1973)

9. Gel'fand,I., Levitan, B. : Am. Math. Soc. Trans. 1, 253-304 (1955)

10. Aronszajn, N.: Am. J. Math. 79, 597-610 (1957)

11. Coddington, E., Levinson, N.: Theory of ordinary differential equations. New York: McGraw-Hill 1955

12. Halmos,P.: Measure theory. Princeton: Van Norstrand 1950

13. Cox,D., Miller,H.: The theory of stochastic processes. London: Methuen 1965

14. Bartle, R.: The elements of integration. New York: Wiley 1966

15. Pearson,D.: Commun. math. Phys. 40, 125-146 (1975)

Communicated by J. Ginibre

Received January 16, 1978 